\title{
Compressed Sensing PARALIND Decomposition-Based Coherent Angle Estimation for Uniform Rectangular Array
}

\author{
Wu Wei, ${ }^{1}$ Xu Le, ${ }^{1}$ Zhang Xiaofei $\left(\mathbb{D},{ }^{1}\right.$ and Li Jianfeng $\mathbb{D}^{1,2}$ \\ ${ }^{1}$ College of Electronic and Information Engineering, Nanjing University of Aeronautics and Astronautics, Nanjing 211106, China \\ ${ }^{2}$ College of Computer and Information, Hohai University, Nanjing 211100, China \\ Correspondence should be addressed to Li Jianfeng; lijianfengtin@126.com
}

Received 17 November 2018; Revised 27 March 2019; Accepted 27 May 2019; Published 24 June 2019

Guest Editor: Alex da Silva

Copyright (c) $2019 \mathrm{Wu}$ Wei et al. This is an open access article distributed under the Creative Commons Attribution License, which permits unrestricted use, distribution, and reproduction in any medium, provided the original work is properly cited.

\begin{abstract}
In this paper, the topic of coherent two-dimensional direction of arrival (2D-DOA) estimation is investigated. Our study jointly utilizes the compressed sensing (CS) technique and the parallel profiles with linear dependencies (PARALIND) model and presents a 2D-DOA estimation algorithm for coherent sources with the uniform rectangular array. Compared to the traditional PARALIND decomposition, the proposed algorithm owns lower computational complexity and smaller data storage capacity due to the process of compression. Besides, the proposed algorithm can obtain autopaired azimuth angles and elevation angles and can achieve the same estimation performance as the traditional PARALIND, which outperforms some familiar algorithms presented for coherent sources such as the forward backward spatial smoothing-estimating signal parameters via rotational invariance techniques (FBSSESPRIT) and forward backward spatial smoothing-propagator method (FBSS-PM). Extensive simulations are provided to validate the effectiveness of the proposed CS-PARALIND algorithm.
\end{abstract}

\section{Introduction}

Array signal processing has aroused considerable concerns in recent decades owing to its extensive engineering application in satellite communication, radar, sonar, and some other fields [1-4]. In array signal processing, spectrum estimation, also known as direction of arrival (DOA) estimation, is a crucial issue. Till now, there are already many neoteric algorithms [5-8] proposed for DOA estimation with linear array, which include estimating signal parameters via rotational invariance techniques (ESPRIT) algorithm [5, 6], multiple signal classification (MUSIC) algorithm [7], and propagator method (PM) [8]. Compared with linear array, rectangular array can measure both azimuth angle and elevation angle, and hence 2D-DOA estimation with rectangular array has motivated enormous investigations. Many traditional DOA estimation methods have already been extended to 2D-DOA estimation [9-12]. For example, the 2D-ESPRIT algorithms [9-11] have utilized the invariance property to obtain the 2DDOA estimation with uniform rectangular array, and the 2DMUSIC [12] algorithm has also proved to be applicable. Some other algorithms such as angle estimation with generalized coprime planar array [13] and 2D-DOA estimation with nested subarrays [14] have better performance for 2D-DOA estimation as well.

However, in many practical situations, the complex propagation environment usually leads to the presence of coherent signals, which makes it complicated to obtain the valid DOA estimation. Therefore, the research on coherent angle estimation has gained great significance. The algorithms mentioned above are only applicable to noncoherent sources and the coherent sources will lead to severe invalidity for these methods. Some other coherent estimation methods including traditional forward spatial smoothing (FSS) or forward backward spatial smoothing (FBSS) $[15,16]$ algorithm have good estimation performance. Exploiting coprime multiple-input multiple-output (MIMO) radar, [17] has proposed the DOA estimation method for mixed coherent and uncorrelated targets and [18] obtained the DOA estimation of coherent sources via fourth-order cumulants.

The trilinear decomposition, namely, parallel factor (PARAFAC) technique [19-21], has been widely employed to resolve the problem of angular estimation with rectangular array [22]. However, the decomposition of PARAFAC model 
fails to work if it contains coherent sources. The parallel profiles with linear dependencies (PARALIND) model [23, 24] discussed in this paper can be regarded as a generalization of PARAFAC model and is efficient to solve the problem of coherent DOA estimation, where the PARAFAC method usually cannot present significant results. In $[25,26]$, the PARALIND decomposition method has already been successfully applied to obtain the coherent sources estimation with MIMO radar and acoustic vector-sensor array. However, the traditional algorithms based on PARALIND decomposition involve heavy computational burden and huge capacity consumption of data storage.

Compressed sensing (CS) $[27,28]$ has aroused considerable concern, which is introduced to areas including channel estimation, image, beamforming, and radar [2932]. Specifically, the angular information of sources can be structured as a sparse vector and hence the CS technique can be directly utilized [33, 34]. By applying CS theory, many novel parameter estimation algorithms have been proposed for different scenarios. Reference [35] proposed a CS-based angle estimation method for noncircular sources which can be applied to MIMO radar, while [33, 34] combined the CS technique with PARAFAC model and proposed an estimation algorithm for joint angle and frequency. Based on the CS PARAFAC model, a 2D-DOA estimation method was presented in [36] with a uniform rectangular array, whereas it works only for noncoherent sources.

In this paper, we propose a CS-PARALIND algorithm for coherent sources to extract the DOA estimates with a uniform rectangular array by generalizing the work presented in [36] and the CS theory for PARALIND decomposition. We first construct the received data model which can be transformed to the PARALIND model. Then, we compress the data model and perform the PARALIND decomposition on it to achieve the estimation of compressed direction matrices. Finally, to acquire the 2D-DOA estimation, we formulate a sparse recovery problem which can be solved by the orthogonal matching pursuit (OMP) method [37]. Note that the compression process only compresses the directional matrix and the source matrix while the coherent matrix remains the same. Therefore, the coherent structure of impinging signals is not destroyed after compression. Comparing to [36], we summarize the main contributions of our research in this paper:

(1) We construct the received data model of coherent signals for uniform rectangular array which is suitable for the PARALIND decomposition.

(2) We generalize the method in [36] and propose the compressed sensing PARALIND (CS-PARALIND) model. Notably, there is no existing report for CS-PARALIND decomposition so far as we know.

(3) We develop the CS-PARALIND decomposition method to obtain the coherent 2D-DOA estimation for uniform rectangular array and give the detailed description of it.

In this paper, the proposed algorithm can obtain autopaired 2D-DOA estimation of coherent signals. In addition, the corresponding correlated matrix can also be

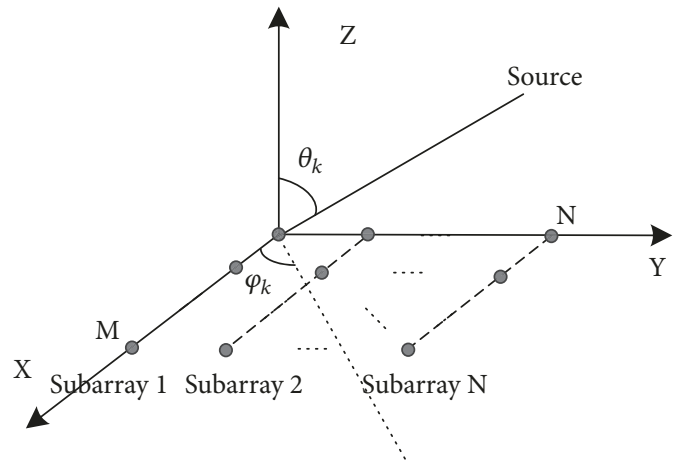

FIGURE 1: The structure of uniform rectangular array.

obtained. The proposed algorithm can attain the same angle estimation performance as the traditional PARALIND method [25]. Compared to the FBSS-ESPRIT and the FBSSPM algorithm, our method can achieve better DOA estimation performance. In addition, due to the process of compression, the proposed algorithm consumes lower computational burden and requires limited storage capacity in practical application. The Cramér-Rao bound (CRB) for the DOA estimation with uniform rectangular array is also provided in this paper. A series of simulation results verify the effectiveness of our approach.

The remainder of our paper is organized as follows: Section 2 presents the received data model of coherent signals with uniform rectangular array. Section 3 depicts the detailed derivation of the proposed CS-PARALIND algorithm as well as the uniqueness certification and complexity analysis. Numerical simulations are exhibited in Section 4, and we conclude this paper in Section 5.

Notation. $\oplus$, ०, and $\otimes$, respectively, represent Hadamard product, Khatri-Rao product, and Kronecker product. (. $)^{H}$, $(.)^{T},(.)^{*},(.)^{-1}$, and $(.)^{+}$stand for the operations of conjugatetranspose, transpose, conjugation, inverse, and pseudoinverse. The Frobenius norm and $l_{0}$-norm are denoted by $\|$ • $\|_{F}$ and $\|\cdot\|_{0} \cdot \operatorname{diag}(\mathbf{b})$ is a diagonal matrix composed of elements in vector $\mathbf{b}$ while $\operatorname{diag}^{-1}(\mathbf{A})$ produces a column vector composed of the diagonal elements of matrix A. $D_{n}(\mathbf{A})$ denotes a diagonal matrix which consists of the $n$-th row of $\mathbf{A}$. $\operatorname{span}(\mathbf{H})$ represents the subspace spanned by the columns of $\mathbf{H}$. $\operatorname{vec}(\mathbf{A})$ means stacking the columns of matrix $\mathbf{A}$. $\partial$ means partial derivatives.

\section{Data Model}

Assume that there are $K$ far-field narrow-band signals with DOA $\left(\theta_{k}, \varphi_{k}\right)$ impinging on a uniform rectangular array consisting of $M \times N$ sensors, where $\varphi_{k}$ is the azimuth angle of the $k$-th signal, $\theta_{k}$ represents the elevation angle, and the distance between any two adjacent elements is $d$. The sources number $K$ is known and the noise is additive white Gaussian which is assumed to be independent and uncorrelated with received signals. Figure 1 shows the structure of the array. 
For the first subarray in the uniform rectangular array, the received data at $t$-th time can be represented as [36]

$$
\mathbf{x}_{1}(t)=\mathbf{A}_{x} \mathbf{s}_{0}(t)+\mathbf{n}_{1}(t)
$$

where $\mathbf{A}_{x}=\left[\mathbf{a}_{x}\left(\theta_{1}, \varphi_{1}\right), \mathbf{a}_{x}\left(\theta_{2}, \varphi_{2}\right), \cdots, \mathbf{a}_{x}\left(\theta_{K}, \varphi_{K}\right)\right]$ denotes the directional matrix of the first subarray with $\mathbf{a}_{x}\left(\theta_{k}, \varphi_{k}\right)=\left[1, \exp \left(j 2 \pi d \cos \varphi_{k} \sin \theta_{k} / \lambda\right), \cdots, \exp (j 2 \pi(M-\right.$ 1) $\left.\left.d \cos \varphi_{k} \sin \theta_{k} / \lambda\right)\right]^{T}$ and $j=\sqrt{-1}$. The received noise of the first subarray is denoted by $\mathbf{n}_{1}(t)$, and $\mathbf{s}_{0}(t)$ is the $K \times 1$ source vector of the $K$ signals. Then it follows that the received data of $n$-th subarray at $t$-th time can be written as [36]

$$
\mathbf{x}_{n}(t)=\mathbf{A}_{x} \boldsymbol{\Phi}^{n-1} \mathbf{s}_{0}(t)+\mathbf{n}_{n}(t),
$$

where $\Phi=\operatorname{diag}\left(\mathrm{e}^{\mathrm{j} 2 \pi d \sin \theta_{1} \sin \varphi_{1} / \lambda}, \cdots, \mathrm{e}^{\mathrm{j} 2 \pi d \sin \theta_{K} \sin \varphi_{K} / \lambda}\right)$ and $\mathbf{n}_{n}(t)$ stands for the received noise of the $n$-th subarray. Therefore, the received data of the entire rectangular array can be denoted by [38]

$$
\mathbf{x}(t)=\left[\begin{array}{c}
\mathbf{x}_{1}(t) \\
\mathbf{x}_{2}(t) \\
\vdots \\
\mathbf{x}_{N}(t)
\end{array}\right]=\left[\begin{array}{c}
\mathbf{A}_{x} \\
\mathbf{A}_{x} \boldsymbol{\Phi} \\
\vdots \\
\mathbf{A}_{x} \boldsymbol{\Phi}^{N-1}
\end{array}\right] \mathbf{s}_{0}(t)+\left[\begin{array}{c}
\mathbf{n}_{1}(t) \\
\mathbf{n}_{2}(t) \\
\vdots \\
\mathbf{n}_{N}(t)
\end{array}\right],
$$

or more compactly as

$$
\mathbf{x}(t)=\left(\mathbf{A}_{y} \circ \mathbf{A}_{x}\right) \mathbf{s}_{0}(t)+\mathbf{n}(t),
$$

where $\mathbf{A}_{y}=\left[\mathbf{a}_{y}\left(\theta_{1}, \varphi_{1}\right), \mathbf{a}_{y}\left(\theta_{2}, \varphi_{2}\right), \cdots, \mathbf{a}_{y}\left(\theta_{K}, \varphi_{K}\right)\right]$ with $\mathbf{a}_{y}\left(\theta_{k}, \varphi_{k}\right)=\left[1, \exp \left(j 2 \pi d \sin \varphi_{k} \sin \theta_{k} / \lambda\right), \cdots, \exp (j 2 \pi(N-\right.$ 1) $\left.\left.d \sin \varphi_{k} \sin \theta_{k} / \lambda\right)\right]$ and $\mathbf{n}(t)$ is the noise of the whole rectangular array with $\mathbf{n}(t)=\left[\mathbf{n}_{1}(t)^{T}, \mathbf{n}_{2}(t)^{T}, \ldots, \mathbf{n}_{N}(t)^{T}\right]^{T}$.

By exploiting $J$ snapshots, the received data can be denoted as [36]

$$
\widetilde{\mathbf{X}}=\left[\mathbf{x}\left(t_{1}\right), \mathbf{x}\left(t_{2}\right), \cdots, \mathbf{x}\left(t_{J}\right)\right],
$$

where $\widetilde{\mathbf{X}}$ stands for the noisy received signals, and rewriting (5) in matrix form, we obtain [38]

$$
\begin{aligned}
\widetilde{\mathbf{X}} & =\left[\begin{array}{c}
\widetilde{\mathbf{X}}_{1} \\
\widetilde{\mathbf{X}}_{2} \\
\vdots \\
\widetilde{\mathbf{X}}_{N}
\end{array}\right]=\left[\begin{array}{c}
\mathbf{A}_{x} D_{1}\left(\mathbf{A}_{y}\right) \\
\mathbf{A}_{x} D_{2}\left(\mathbf{A}_{y}\right) \\
\vdots \\
\mathbf{A}_{x} D_{N}\left(\mathbf{A}_{y}\right)
\end{array}\right] \mathbf{S}_{0}+\left[\begin{array}{c}
\mathbf{N}_{1} \\
\mathbf{N}_{2} \\
\vdots \\
\mathbf{N}_{N}
\end{array}\right] \\
& =\left[\mathbf{A}_{y} \circ \mathbf{A}_{x}\right] \mathbf{S}_{0}+\mathbf{N},
\end{aligned}
$$

where $\mathbf{S}_{0}=\left[\mathbf{s}_{0}\left(t_{1}\right), \mathbf{s}_{0}\left(t_{2}\right), \cdots, \mathbf{s}_{0}\left(t_{J}\right)\right] \in \mathbf{C}^{K \times J}$ and $\widetilde{\mathbf{X}}_{n}=$ $\mathbf{A}_{x} D_{n}\left(\mathbf{A}_{y}\right) \mathbf{S}_{0}+\mathbf{N}_{n}$. The noise of $J$ snapshots is $\mathbf{N}=$ $\left[\mathbf{n}\left(t_{1}\right), \mathbf{n}\left(t_{2}\right), \cdots, \mathbf{n}\left(t_{J}\right)\right] \in \mathbf{C}^{M N \times J}$.

The received data matrix shown in (6) is a traditional PARAFAC model [19-22], the decomposition of which is usually nonunique if there exist coherent signals [19].
Assume that among the $K$ received sources, there are $K_{1}$ groups of coherent signals; then (6) can be represented as [25]

$$
\widetilde{\mathbf{X}}=\left[\mathbf{A}_{y} \circ \mathbf{A}_{x}\right] \Gamma \mathbf{S}+\mathbf{N},
$$

where $\mathbf{S} \in \mathbf{C}^{K_{1} \times J}$ is the source matrix of $J$ snapshots of $K_{1}$ noncoherent signals. $\Gamma \in \mathbf{C}^{K \times K_{1}}$ is the corresponding correlated matrix satisfying $\Gamma S=S_{0}$.

\section{CS-PARALIND Decomposition-Based Algorithm}

The PARALIND algorithm suffers from expensive computational cost, especially in the case of large number of sensors or snapshots. Specifically, the angular information of received sources can be structured as a sparse vector and hence the CS technique can be directly utilized [33]. To counter this problem, we bring in the CS theory by compressing the received signal in (7) to a smaller matrix, followed by the PARALIND decomposition and, finally, acquire the 2D-DOA estimates by exploiting sparse recovery method.

3.1. Compression. We compress the received data $\underline{\mathbf{X}} \in$ $\mathbf{C}^{M \times J \times N}$ into a smaller matrix $\underline{\mathbf{X}^{\prime}} \in \mathbf{C}^{M^{\prime} \times J^{\prime} \times N^{\prime}}$ with three compression matrices $\mathbf{U} \in \mathbf{C}^{M \times M^{\prime}}, \mathbf{V} \in \mathbf{C}^{N \times N^{\prime}}$, and $\mathbf{W} \in$ $\mathrm{C}^{J \times J^{\prime}}$, with $M^{\prime}<M, J^{\prime}<J$ and $N^{\prime}<N$, which is illustrated in Figure 2.

The three compression matrices can be constructed via some random special matrices, e.g., the random Gaussian, Bernoulli, and partial Fourier matrices or the Tucker3 decomposition introduced in $[33,39]$.

Applying $\mathbf{U}, \mathbf{V}$, and $\mathbf{W}$ to (7), we can obtain the compressed received data $\widetilde{\mathbf{X}}^{\prime} \in \mathbf{C}^{\left(M^{\prime} N^{\prime} \times J^{\prime}\right)}$ as [27]

$$
\begin{aligned}
\widetilde{\mathbf{X}}^{\prime} & =\left(\mathbf{V}^{T} \otimes \mathbf{U}^{T}\right) \widetilde{\mathbf{X}}^{(M N \times J)} \mathbf{W} \\
& =\left(\mathbf{V}^{T} \otimes \mathbf{U}^{T}\right)\left[\mathbf{A}_{y} \circ \mathbf{A}_{x}\right] \mathbf{r S W}+\left(\mathbf{V}^{T} \otimes \mathbf{U}^{T}\right) \mathbf{N W} .
\end{aligned}
$$

And according to the property of Khatri-Rao product [33], we have $\left(\mathbf{V}^{T} \otimes \mathbf{U}^{T}\right)\left(\mathbf{A}_{y} \circ \mathbf{A}_{x}\right)=\left(\mathbf{V}^{T} \mathbf{A}_{y}\right) \circ\left(\mathbf{U}^{T} \mathbf{A}_{x}\right)$, and then (8) can be further simplified to

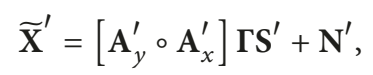

where $\mathbf{A}_{x}^{\prime\left(M^{\prime} \times K\right)}=\mathbf{U}^{T} \mathbf{A}_{x}, \mathbf{A}_{x}^{\prime\left(M^{\prime} \times K\right)}=\mathbf{U}^{T} \mathbf{A}_{x}, \mathbf{A}_{y}^{\prime\left(N^{\prime} \times K\right)}=$ $\mathbf{V}^{T} \mathbf{A}_{y}, \mathbf{S}^{\prime\left(K \times J^{\prime}\right)}=\mathbf{S W}$, and $\mathbf{N}^{\prime}=\left(\mathbf{V}^{T} \otimes \mathbf{U}^{T}\right) \mathbf{N W}$.

3.2. PARALIND Decomposition. To transform (9) to PARALIND model, we define $\widetilde{\mathbf{Y}}=\widetilde{\mathbf{X}}^{\prime T}$, or equivalently,

$$
\widetilde{\mathbf{Y}}=\left(\boldsymbol{\Gamma} \mathbf{S}^{\prime}\right)^{T}\left(\mathbf{A}_{y}^{\prime} \circ \mathbf{A}_{x}^{\prime}\right)^{T}+\mathbf{N}^{T}=\mathbf{Y}+\mathbf{N}^{T},
$$

where $\mathbf{Y}=\left(\boldsymbol{\Gamma S}^{\prime}\right)^{T}\left(\mathbf{A}_{y}^{\prime} \circ \mathbf{A}_{x}^{\prime}\right)^{T}$ denotes the noise-free compressed received matrix. 


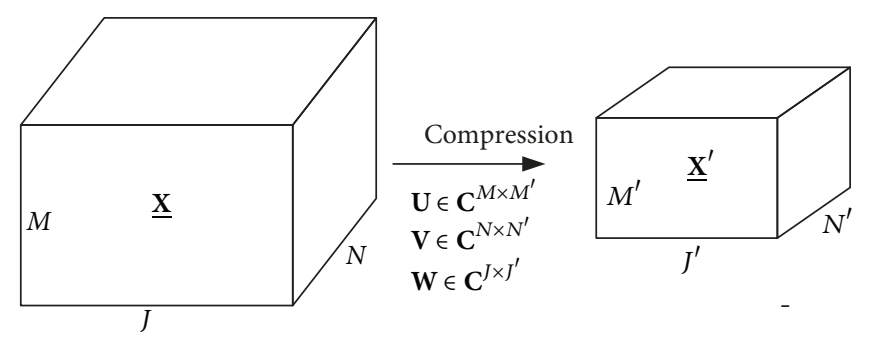

FIgURE 2: Compression processing.

Obviously, (10) is equivalent to the PARALIND model [20] and the least fitting for it amounts to [23]

$$
\min _{\Gamma, \mathbf{S}, \mathbf{A}_{y}^{\prime}, \mathbf{A}_{x}^{\prime}}\left\|\widetilde{\mathbf{Y}}-\left(\mathbf{\Gamma} \mathbf{S}^{\prime}\right)^{T}\left(\mathbf{A}_{y}^{\prime} \circ \mathbf{A}_{x}^{\prime}\right)^{T}\right\|_{F}^{2} .
$$

Under noise-free condition, (10) can be rewritten as

$$
\begin{aligned}
\mathbf{Y} & =\left[\mathbf{Y}_{1}, \mathbf{Y}_{2}, \cdots, \mathbf{Y}_{N^{\prime}}\right]=\left[\left(\mathbf{\Gamma} \mathbf{S}^{\prime}\right)^{T} D_{1}\left(\mathbf{A}_{y}^{\prime}\right) \mathbf{A}_{x}^{\prime T},\left(\mathbf{\Gamma} \mathbf{S}^{\prime}\right)^{T}\right. \\
& \left.\cdot D_{2}\left(\mathbf{A}_{y}^{\prime}\right) \mathbf{A}_{x}^{\prime T}, \cdots,\left(\boldsymbol{\Gamma} \mathbf{S}^{\prime}\right)^{T} D_{N^{\prime}}\left(\mathbf{A}_{y}^{\prime}\right) \mathbf{A}_{x}^{\prime T}\right]
\end{aligned}
$$

where $\mathbf{Y}_{n}=\left(\boldsymbol{\Gamma} \mathbf{S}^{\prime}\right)^{T} D_{n}\left(\mathbf{A}_{y}^{\prime}\right) \mathbf{A}_{x}^{\prime T} \in \mathbf{C}^{I^{\prime} \times M^{\prime}}, n=1,2, \cdots, N^{\prime}$.

After vectoring $\mathbf{Y}_{n}$, we have [38]

$$
\begin{aligned}
\operatorname{vec}\left(\mathbf{Y}_{n}\right) & =\operatorname{vec}\left(\left(\mathbf{\Gamma} \mathbf{S}^{\prime}\right)^{T} D_{n}\left(\mathbf{A}_{y}^{\prime}\right) \mathbf{A}_{x}^{\prime T}\right) \\
& =\left(\mathbf{A}_{x}^{\prime} D_{n}\left(\mathbf{A}_{y}^{\prime}\right) \otimes \mathbf{S}^{\prime T}\right) \operatorname{vec}\left(\mathbf{\Gamma}^{T}\right) .
\end{aligned}
$$

Stacking these vectors by columns

$$
\left[\begin{array}{c}
\operatorname{vec}\left(\mathbf{Y}_{1}\right) \\
\operatorname{vec}\left(\mathbf{Y}_{2}\right) \\
\vdots \\
\operatorname{vec}\left(\mathbf{Y}_{N^{\prime}}\right)
\end{array}\right]=\left[\begin{array}{c}
\mathbf{A}_{x}^{\prime} D_{1}\left(\mathbf{A}_{y}^{\prime}\right) \otimes \mathbf{S}^{\prime T} \\
\mathbf{A}_{x}^{\prime} D_{2}\left(\mathbf{A}_{y}^{\prime}\right) \otimes \mathbf{S}^{\prime T} \\
\vdots \\
\mathbf{A}_{x}^{\prime} D_{N^{\prime}}\left(\mathbf{A}_{y}^{\prime}\right) \otimes \mathbf{S}^{\prime T}
\end{array}\right] \operatorname{vec}\left(\boldsymbol{\Gamma}^{T}\right)
$$

Equation (14) also can be denoted as

$$
\operatorname{vec}(\mathbf{Y})=\left[\left(\mathbf{A}_{y}^{\prime} \circ \mathbf{A}_{x}^{\prime}\right) \otimes \mathbf{S}^{\prime T}\right] \operatorname{vec}\left(\mathbf{\Gamma}^{T}\right) .
$$

Then under noise environment, $\operatorname{vec}\left(\boldsymbol{\Gamma}^{T}\right)$ can be obtained by

$$
\operatorname{vec}\left(\mathbf{\Gamma}^{T}\right)=\left[\left(\mathbf{A}_{y}^{\prime} \circ \mathbf{A}_{x}^{\prime}\right) \otimes \mathbf{S}^{T}\right]^{+} \operatorname{vec}(\widetilde{\mathbf{Y}}) .
$$

Now we can update the value of $\boldsymbol{\Gamma}$ via transforming $\operatorname{vec}\left(\boldsymbol{\Gamma}^{T}\right)$ to its original modality.

According to (11), the least square (LS) update for $\mathbf{S}^{\prime T}$ is

$$
\mathbf{S}^{T}=\widetilde{\mathbf{Y}}\left(\left(\left(\mathbf{A}_{x}^{\prime} \circ \mathbf{A}_{y}^{\prime}\right) \mathbf{\Gamma}\right)^{T}\right)^{+} .
$$

From (12), it follows that the covariance matrix of $\mathbf{Y}_{n}$ can be written as

$$
\begin{aligned}
& \sum_{n=1}^{N^{\prime}} \mathbf{Y}_{n}{ }^{H} \mathbf{Y}_{n}=\left(\sum_{n=1}^{N^{\prime}} \mathbf{Y}_{n}{ }^{H}\left(\boldsymbol{\Gamma} \mathbf{S}^{\prime}\right)^{T} D_{n}\left(\mathbf{A}_{y}^{\prime}\right)\right) \mathbf{A}_{x}^{\prime T} \\
& =\mathbf{A}_{x}^{\prime *}\left(\sum_{n=1}^{N^{\prime}} D_{n}{ }^{*}\left(\mathbf{A}_{y}^{\prime}\right)\left(\boldsymbol{\Gamma} \mathbf{S}^{\prime}\right)^{*}\left(\boldsymbol{\Gamma} \mathbf{S}^{\prime}\right)^{T} D_{n}\left(\mathbf{A}_{y}^{\prime}\right)\right) \mathbf{A}_{x}^{\prime T} .
\end{aligned}
$$

As $\mathbf{A}_{x}^{\prime}$ is of full column rank and $\sum_{n=1}^{N^{\prime}} D_{n}{ }^{*}\left(\mathbf{A}_{y}^{\prime}\right)\left(\mathbf{\Gamma} \mathbf{S}^{\prime}\right)^{*}\left(\mathbf{\Gamma} \mathbf{S}^{\prime}\right)^{T} D_{n}\left(\mathbf{A}_{y}^{\prime}\right)$ is nonsingular [24], we can obtain $\mathbf{A}_{x}^{\prime *}$ via

$$
\begin{aligned}
& \mathbf{A}_{x}^{\prime *}=\left(\sum_{n=1}^{N^{\prime}} \widetilde{\mathbf{Y}}_{n}^{H}\left(\boldsymbol{\Gamma} \mathbf{S}^{\prime}\right)^{T} D_{n}\left(\mathbf{A}_{y}^{\prime}\right)\right) \\
& \cdot\left(\sum_{n=1}^{N^{\prime}} D_{n}^{*}\left(\mathbf{A}_{y}^{\prime}\right)\left(\boldsymbol{\Gamma} \mathbf{S}^{\prime}\right)^{*}\left(\boldsymbol{\Gamma} \mathbf{S}^{\prime}\right)^{T} D_{n}\left(\mathbf{A}_{y}^{\prime}\right)\right)^{-1} \\
& =\left(\sum_{n=1}^{N^{\prime}} \widetilde{\mathbf{Y}}_{n}^{H}\left(\mathbf{\Gamma} \mathbf{S}^{\prime}\right)^{T} D_{n}\left(\mathbf{A}_{y}^{\prime}\right)\right) \\
& \cdot\left(\sum_{n=1}^{N^{\prime}}\left(\left(\boldsymbol{\Gamma} \mathbf{S}^{\prime}\right)^{*}\left(\mathbf{\Gamma} \mathbf{S}^{\prime}\right)^{T}\right) \oplus\left(\mathbf{A}_{y}^{\prime H} \mathbf{A}_{y}^{\prime}\right)\right)^{-1} \text {. }
\end{aligned}
$$

Substitute $\mathbf{Y}_{n}$ of (12) into the following

$$
\left(\boldsymbol{\Gamma} \mathbf{S}^{\prime}\right)^{*} \mathbf{Y}_{n} \mathbf{A}_{x}^{\prime}=\left(\boldsymbol{\Gamma} \mathbf{S}^{\prime}\right)^{*}\left(\boldsymbol{\Gamma} \mathbf{S}^{\prime}\right)^{T} D_{n}\left(\mathbf{A}_{y}^{\prime}\right) \mathbf{A}_{x}^{\prime T} \mathbf{A}_{x}^{\prime} .
$$

Taking the diagonal elements of both sides in (20), we have

$$
\begin{aligned}
\operatorname{diag}^{-1} & \left(\left(\boldsymbol{\Gamma} \mathbf{S}^{\prime}\right)^{*} \mathbf{Y}_{n} \mathbf{A}_{x}^{\prime}\right) \\
= & \left(\left(\mathbf{\Gamma} \mathbf{S}^{\prime}\right)^{*}\left(\mathbf{\Gamma} \mathbf{S}^{\prime}\right)^{T}\right) \\
& \oplus\left(\mathbf{A}_{x}^{\prime T} \mathbf{A}_{x}^{\prime}\right) \operatorname{diag}^{-1}\left(D_{n}\left(\mathbf{A}_{y}^{\prime}\right)\right) .
\end{aligned}
$$


By enforcing left multiplication operation of (21), we get

$$
\begin{aligned}
& \operatorname{diag}^{-1}\left(D_{n}\left(\mathbf{A}_{y}^{\prime}\right)\right)=\left(\left(\left(\boldsymbol{\Gamma} \mathbf{S}^{\prime}\right)^{*}\left(\mathbf{\Gamma} \mathbf{S}^{\prime}\right)^{T}\right)\right. \\
& \left.\oplus\left(\mathbf{A}_{x}^{\prime T} \mathbf{A}_{x}^{\prime}\right)\right)^{-1} \operatorname{diag}^{-1}\left(\left(\boldsymbol{\Gamma} \mathbf{S}^{\prime}\right)^{*} \widetilde{\mathbf{Y}}_{n} \mathbf{A}_{x}^{\prime}\right) .
\end{aligned}
$$

And hence, $\mathbf{A}_{y}$ can be easily obtained form (22).

For the PARALIND decomposition above, according to (17), (18), and (19), we repeatedly update the estimation of $\mathbf{A}_{x}^{\prime}, \mathbf{A}_{y}^{\prime}, \mathbf{S}^{\prime}$, and $\boldsymbol{\Gamma}$ until convergence. Define the sum of squared residuals (SSR) of the $k$-th iteration as $S S R_{k}=$ $\sum_{i=1}^{N^{\prime} M^{\prime}} \sum_{j=1}^{J^{\prime}}\left|c_{i j}\right|^{2}$, where $c_{i j}$ is the $(i, j)$ element of $\mathbf{C}=\tilde{\mathbf{Y}}-$ $\left(\widehat{\boldsymbol{\Gamma}} \widehat{\mathbf{S}}^{\prime}\right)^{T}\left(\widehat{\mathbf{A}}_{y}^{\prime} \circ \widehat{\mathbf{A}}_{x}^{\prime}\right)^{T}$. Define the convergence rate of PARALIND decomposition as $S S R_{\text {rate }}=\left(S S R_{k}-S S R_{k-1}\right) / S S R_{k-1}[40]$. When $S S R_{\text {rate }}$ is smaller than a certain small value (determined by the noise level), then the above iteration process can be considered convergent.

Based on the PARALIND decomposition, the estimates of $\mathbf{A}_{x}^{\prime}$ and $\mathbf{A}_{y}^{\prime}$ can be achieved by

$$
\begin{aligned}
& \widehat{\mathbf{A}}_{x}^{\prime}=\mathbf{A}_{x}^{\prime} \Pi \Delta_{x}+\mathbf{W}_{x}=\mathbf{U}^{T} \mathbf{A}_{x} \Pi \Delta_{x}+\mathbf{W}_{x}, \\
& \widehat{\mathbf{A}}_{y}^{\prime}=\mathbf{A}_{y}^{\prime} \boldsymbol{\Pi} \Delta_{y}+\mathbf{W}_{y}=\mathbf{V}^{T} \mathbf{A}_{y} \boldsymbol{\Pi} \Delta_{y}+\mathbf{W}_{y},
\end{aligned}
$$

where $\Pi$ stands for the permutation matrix and $\Delta_{y}$ and $\Delta_{x}$ are the diagonal scaling matrix of $\mathbf{A}_{y}^{\prime}$ and $\mathbf{A}_{x}^{\prime}$. In addition, $\mathbf{W}_{x}$ and $\mathbf{W}_{y}$ denote the estimation error.

Remark 1 . In the proposed algorithm, the scale ambiguity can be eliminated by direct normalization while the permutation ambiguity makes no difference in the angle estimation.

3.3. DOA Estimation with Sparsity. By utilizing $\widehat{\mathbf{A}}_{x}^{\prime}, \widehat{\mathbf{A}}_{y}^{\prime}$, the 2D-DOA estimation can be obtained with sparsity. With noiseless case, we use $\widehat{\mathbf{a}}_{x k}^{\prime}$ and $\widehat{\mathbf{a}}_{y k}^{\prime}$ to denote the $k$-th column of $\widehat{\mathbf{A}}_{x}^{\prime}, \widehat{\mathbf{A}}_{y}^{\prime}$, respectively. From (23) and (24), it follows that [36]

$$
\begin{aligned}
& \widehat{\mathbf{a}}_{x k}^{\prime}=\mathbf{U}^{H} \rho_{x k} \mathbf{a}_{x k}, \\
& \widehat{\mathbf{a}}_{y k}^{\prime}=\mathbf{V}^{H} \rho_{y k} \mathbf{a}_{y k},
\end{aligned}
$$

where $\mathbf{a}_{x k}$ and $\mathbf{a}_{y k}$ represent the $k$-th column in the directional matrices $\mathbf{A}_{x}$ and $\mathbf{A}_{y}$. In addition, $\rho_{x k}$ and $\rho_{y k}$ denote the scaling coefficients.

Then we construct two Vandermonde matrices $\mathbf{A}_{s x} \in$ $\mathbf{C}^{M \times Q}$ and $\mathbf{A}_{s y} \in \mathbf{C}^{N \times Q}(Q>>M, Q>>N)$, the columns of which consist of the steering vectors corresponding to each potential source location [36].

$$
\begin{aligned}
& \mathbf{A}_{s x}=\left[\mathbf{a}_{s x 1}, \mathbf{a}_{s x 2}, \cdots, \mathbf{a}_{s x Q}\right] \\
& =\left[\begin{array}{cccc}
1 & 1 & \cdots & 1 \\
e^{j 2 \pi d \mathbf{g}(1) / \lambda} & e^{j 2 \pi d \mathbf{g}(2) / \lambda} & \vdots & e^{j 2 \pi d \mathbf{g}(Q) / \lambda} \\
\vdots & \vdots & \vdots & \vdots \\
e^{j 2 \pi(M-1) d \mathbf{g}(1) / \lambda} & e^{j 2 \pi(M-1) d \mathbf{g}(2) / \lambda} & \cdots & e^{j 2 \pi(M-1) d \mathbf{g}(Q) / \lambda}
\end{array}\right], \\
& \mathbf{A}_{s y}=\left[\begin{array}{cccc}
\left.\mathbf{a}_{s y 1}, \mathbf{a}_{s y 2}, \cdots, \mathbf{a}_{s y Q}\right] & & \\
1 & 1 & \cdots & 1 \\
e^{j 2 \pi d \mathbf{g}(1) / \lambda} & e^{j 2 \pi d \mathbf{g}(2) / \lambda} & \vdots & e^{j 2 \pi d \mathbf{g}(Q) / \lambda} \\
\vdots & \vdots & \vdots & \vdots \\
e^{j 2 \pi(N-1) d \mathbf{g}(1) / \lambda} & e^{j 2 \pi(N-1) d \mathbf{g}(2) / \lambda} & \cdots & e^{j 2 \pi(N-1) d \mathbf{g}(Q) / \lambda}
\end{array}\right],
\end{aligned}
$$

where $\mathbf{g}$ is a sampling vector with $\mathbf{g}(q)=-1+2 q / Q, q=$ $0,1, \ldots, Q \cdot \mathbf{A}_{s x}$ and $\mathbf{A}_{s y}$ can be referred to as the overcomplete dictionary for our 2D-DOA estimation [36]. Then (25), (26) can be converted to

$$
\begin{aligned}
& \widehat{\mathbf{a}}_{x k}^{\prime}=\mathbf{U}^{T} \mathbf{A}_{s x} \mathbf{x}_{s k}, \quad k=1, \cdots, K, \\
& \widehat{\mathbf{a}}_{y k}^{\prime}=\mathbf{V}^{T} \mathbf{A}_{s y} \mathbf{y}_{s k}, \quad k=1, \cdots, K,
\end{aligned}
$$

where $\mathbf{x}_{s k}$ and $\mathbf{y}_{s k}$ are sparse, the estimates of which can be cast as an optimization problem, subject to $l_{0}$-norm constraint.

$$
\begin{array}{ll}
\min & \left\|\widehat{\mathbf{a}}_{x k}^{\prime}-\mathbf{U}^{H} \mathbf{A}_{s x} \mathbf{x}_{s k}\right\|_{F}^{2}, \\
\text { st. } & \left\|\mathbf{x}_{s k}\right\|_{0}=1, \\
\min & \left\|\widehat{\mathbf{a}}_{y k}^{\prime}-\mathbf{V}^{H} \mathbf{A}_{s y} \mathbf{y}_{s k}\right\|_{F}^{2}, \\
\text { st. } & \left\|\mathbf{y}_{s k}\right\|_{0}=1 .
\end{array}
$$

$\mathbf{x}_{s k}$ and $\mathbf{y}_{s k}$ can be gained by the OMP recovery method [37].

Denote the maximum modulus of elements in $\mathbf{x}_{s k}$ and $\mathbf{y}_{s k}$ as $q_{x k}$ and $q_{y k}$, respectively. Then the corresponding elements $\mathbf{g}\left(q_{x k}\right)$ and $\mathbf{g}\left(q_{y k}\right)$ in $\mathbf{A}_{s x}$ and $\mathbf{A}_{s y}$ are just the $\sin \theta_{k} \cos \varphi_{k}$ and $\sin \theta_{k} \sin \varphi_{k}$ estimation. Define $r_{k}=\mathbf{g}\left(q_{x k}\right)+j \mathbf{g}\left(q_{y k}\right)$ and the estimates of elevation angles and azimuth angles can be obtained by

$$
\begin{aligned}
& \widehat{\theta}_{k}=\sin ^{-1}\left(\operatorname{abs}\left(r_{k}\right)\right), \quad k=1,2, \ldots, K, \\
& \widehat{\varphi}_{k}=\operatorname{angle}\left(r_{k}\right), \quad k=1,2, \ldots, K,
\end{aligned}
$$

where $a b s($.$) outputs the modulus value and angle(.) com-$ putes the angular part of a complex number. Finally, the autopaired estimates of elevation and azimuth angles can be attained by employing the paired relationship in the directional matrices obtained by PARALIND decomposition.

3.4. The Procedure of the Proposed Algorithm. Till now, the angle estimation of received coherent signals with a uniform rectangular array has been acquired and we provide the major steps as follows: 
Step 1. Compress the received data $\widetilde{\mathbf{X}}$, construct the PARALIND model $\widetilde{\mathbf{Y}}$ according to (8), and then initialize the value of $\mathbf{A}_{x}^{\prime}, \mathbf{A}_{y}^{\prime}, \boldsymbol{\Gamma}$, and $\mathbf{S}^{\prime}$.

Step 2. According to (16), (17), (19), and (22), repeatedly update the estimates of $\mathbf{A}_{x}^{\prime}, \mathbf{A}_{y}^{\prime}, \mathbf{S}^{\prime}$, and $\Gamma$ according to the convergence conditions.

Step 3. According to (31)-(34), the elevation and azimuth angles can be estimated by $\mathbf{A}_{s x}$ and $\mathbf{A}_{s y}$.

\section{Performance Analysis}

4.1. Complexity Analysis of the Proposed Algorithm. We analyze the complexity of the proposed algorithm in this subsection. For the proposed algorithm, the computational complexity is $O\left(M^{\prime} N^{\prime} M N J+J M^{\prime} N^{\prime} J^{\prime}+n_{1}\left(M^{\prime} N^{\prime} J^{\prime}\left(2 K^{2} K_{1}{ }^{2}+\right.\right.\right.$ $\left.2 K K_{1}+2 K+K_{1}\right)+M^{\prime} N^{\prime}\left(2 K_{1}^{2}+K^{2}+K K_{1}+2 K\right)+$ $K^{2}\left(J^{\prime} N^{\prime}+J^{\prime}+N^{\prime 2}+N^{\prime}+M^{\prime}+2\right)+K^{3} K_{1}^{3}+2 K^{3}+$ $\left.\left.\left.K_{1}{ }^{3}+K K_{1} J^{\prime}\left(N^{\prime}+1\right)\right)\right)+K P(M+N)\right)$, where $n_{1}$ denotes the time of iterations. With regard to the traditional PARALIND decomposition [23], it has $\left(n_{2}\left(M N J\left(2 K^{2} K_{1}^{2}+2 K K_{1}+2 K+\right.\right.\right.$ $\left.K_{1}\right)+M N\left(2 K_{1}^{2}+K^{2}+K K_{1}+2 K\right)+K^{2}\left(J N+J+N^{2}+\right.$ $\left.N+M+2)+K^{3} K_{1}{ }^{3}+2 K^{3}+K_{1}{ }^{3}+K K_{1} J(N+1)\right)+$ $\left.2 K^{2}(M+N)+6 K^{2}\right)$, where $n_{2}$ represents the time of iterations. In the comparison of computational complexity, we have the parameters of $M=N=10, K=3$, and $K_{1}=2$. In addition, assume that $n_{1}=n_{2}=20, P=200$, and $M^{\prime} / M=$ $N^{\prime} / N=J^{\prime} / J=0.5$. The comparison of complexity versus $J$ is depicted in Figure 3, which concludes that the computational burden of the proposed algorithm is decreased compared to the traditional PARALIND algorithm.

4.2. CRB. Define $\mathbf{A}=\left[\mathbf{a}_{y}\left(\theta_{1}, \varphi_{1}\right) \otimes\right.$ $\left.\mathbf{a}_{x}\left(\theta_{1}, \varphi_{1}\right), \cdots, \mathbf{a}_{y}\left(\theta_{K}, \varphi_{K}\right) \otimes \mathbf{a}_{x}\left(\theta_{K}, \varphi_{K}\right)\right]$ and according to [41], we derive the CRB

$$
C R B=\frac{\sigma^{2}}{2 J}\left\{\operatorname{Re}\left[\mathbf{D}^{H} \boldsymbol{\Pi}_{\mathbf{A}}^{\perp} \mathbf{D} \oplus \widehat{\mathbf{P}}_{s}^{T}\right]\right\}^{-1},
$$

where $J$ is the number of snapshots and $\mathbf{a}_{k}$ is the $k$ th column of $\mathbf{A}$. $\sigma^{2}$ denotes the noise power. $\boldsymbol{\Pi}_{\mathbf{A}}^{\perp}=$ $\mathbf{I}_{M N}-\mathbf{A}\left(\mathbf{A}^{H} \mathbf{A}\right)^{-1} \mathbf{A}^{H}$ and $\mathbf{I}_{M N}$ is an $M N \times M N$ identity matrix. $\mathbf{D}=\left[\partial \mathbf{a}_{1} / \partial \theta_{1}, \partial \mathbf{a}_{2} / \partial \theta_{2}, \ldots, \partial \mathbf{a}_{K} / \partial \theta_{K}, \partial \mathbf{a}_{1} / \partial \varphi_{1}, \partial \mathbf{a}_{2} / \partial \varphi_{2}, \ldots\right.$, $\left.\partial \mathbf{a}_{K} / \partial \varphi_{K}\right]$ and $\widehat{\mathbf{P}}_{s}=(1 / J) \sum_{t=1}^{J} \mathbf{s}(t) \mathbf{s}^{H}(t)$.

4.3. Advantages. We summarize the advantages of the proposed algorithm as follows:

(1) The proposed algorithm possesses lower computational cost and requires smaller data storage, due to the compression operation.

(2) The proposed algorithm can be applied to the coherent signals. Furthermore, the corresponding correlated matrix also can be obtained.

(3) The proposed algorithm can achieve the same angle estimation performance with the traditional PARALIND method and outperforms the FBSS-ESPRIT and FBSS-PM algorithm.

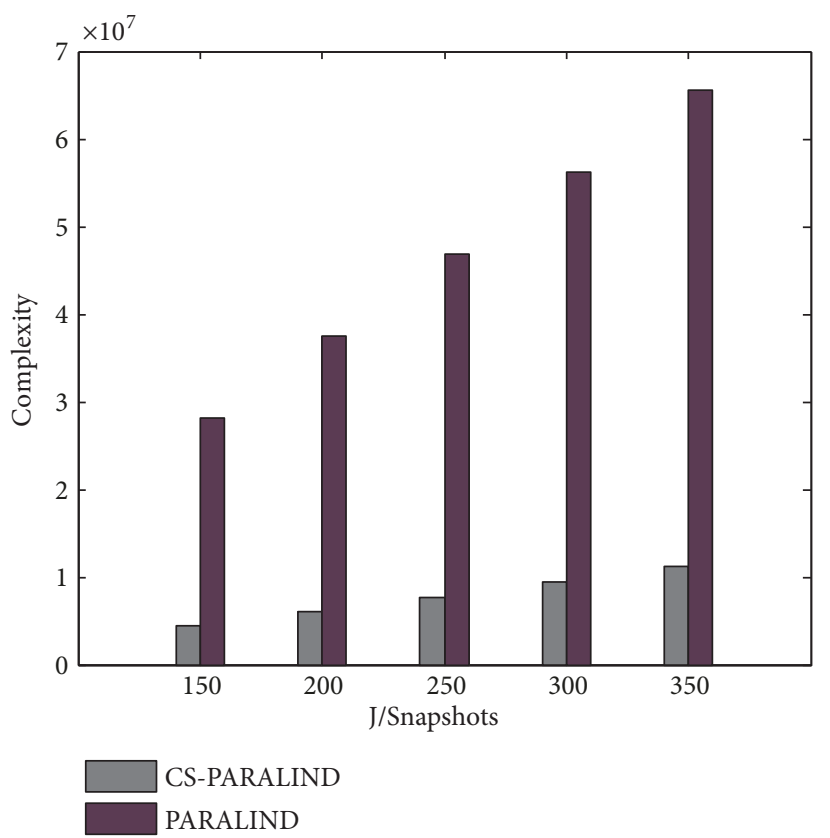

Figure 3: Comparison of complexity versus different snapshots $(J)$.

\section{Simulation Results}

Suppose that $K=3$ signals with two coherent signals and one noncoherent signal impinge on a rectangular array, where the correlated matrix is $\Gamma=\left[\begin{array}{lll}1 & 0 & 1 \\ 0 & 1 & 0\end{array}\right]^{T}$. In the following simulations, we exploit the root mean square error (RMSE) to evaluate the DOA estimation performance and it is presented by

$$
R M S E=\frac{1}{K} \sum_{k=1}^{K} \sqrt{\frac{1}{L} \sum_{l=1}^{L}\left(\widehat{\varphi}_{k, l}-\varphi_{k}\right)^{2}+\left(\widehat{\theta}_{k, l}-\theta_{k}\right)^{2}},
$$

where $\widehat{\varphi}_{k, l}, \widehat{\theta}_{k, l}$ are the estimations of $\varphi_{k}, \theta_{k}$ in $l$-th simulation, and the times of Monte-Carlo simulations are indicated by $L$. In the following simulations, we set $L=1000$.

The locations of the three sources are $\left(\theta_{1}, \varphi_{1}\right)=\left(15^{\circ}, 10^{\circ}\right)$, $\left(\theta_{2}, \varphi_{2}\right)=\left(25^{\circ}, 30^{\circ}\right)$, and $\left(\theta_{3}, \varphi_{3}\right)=\left(35^{\circ}, 50^{\circ}\right) . M, N, J, K$ are the number of rows and columns of the rectangular array, snapshots, and sources, respectively. And assume that the rectangular array is uniform and the distance of any two adjacent sensors is $\lambda / 2$.

Simulation 1. Figure 4 shows the 2D-DOA estimation results of our proposed algorithm with $S N R=0 \mathrm{~dB}$ and $\mathrm{SNR}=20 \mathrm{~dB}$, respectively. The size of PARALIND model is defined as $M \times$ $N \times J$. The received data matrix has the size of $10 \times 10 \times 200$ in this simulation, which becomes $7 \times 7 \times 140\left(M^{\prime} \times N^{\prime} \times J^{\prime}\right)$ after compression. Figure 4 indicates that the proposed algorithm is effective for 2D-DOA estimation of coherent sources.

Simulation 2. In Figure 5, we exhibit the comparison of the DOA estimation performance among the proposed algorithm, FSS-ESPRIT, FSS-PM, FBSS-ESPRIT, and FBSS-PM algorithm, and the traditional PARALIND algorithm. The 


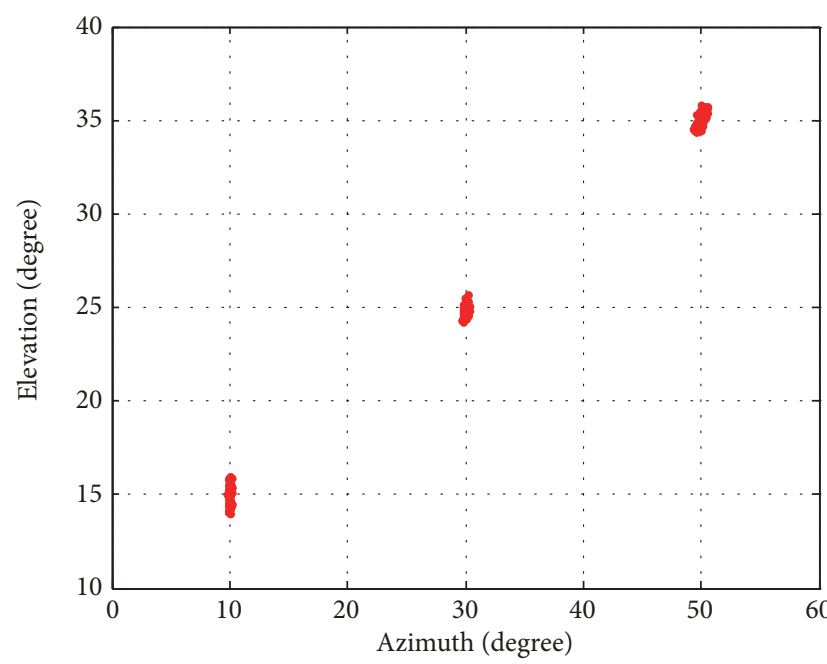

(a)

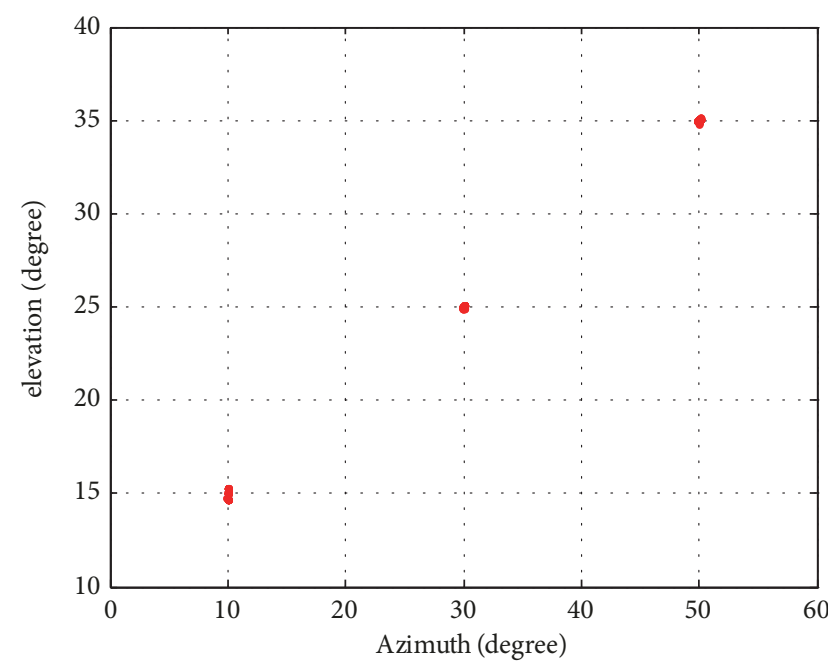

(b)

FIGURE 4: DOA estimation of the proposed algorithm with (a) SNR=0dB, (b) SNR=20dB.

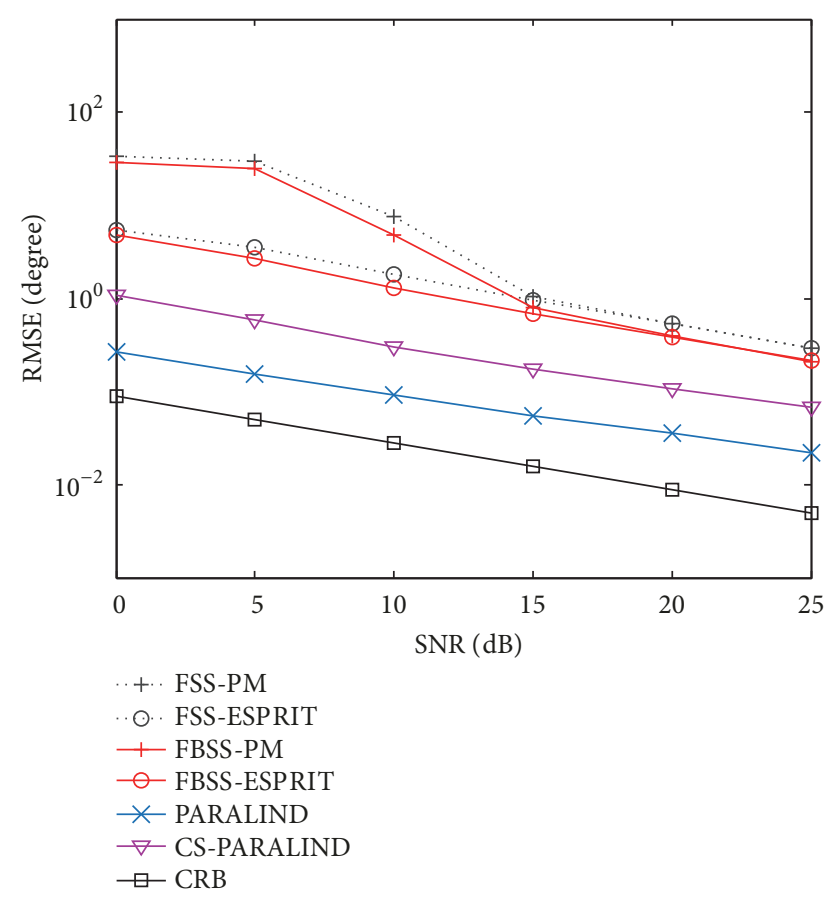

FIGURE 5: DOA estimation performance comparison.

received data matrix has the size of $10 \times 10 \times 100$ and is compressed to the size of $8 \times 8 \times 80$. It is illustrated clearly in Figure 5 that the approach algorithm can achieve the same DOA estimation performance compared with the traditional PARALIND algorithm and, furthermore, outperforms the other four algorithms.

Simulation 3. The DOA estimation performance result of the proposed algorithm versus compression ratio is provided in Figure 6, where the compression ratio is $P=M^{\prime} / M=$ $N^{\prime} / N=J^{\prime} / J$. In this part, the size of the original PARALIND

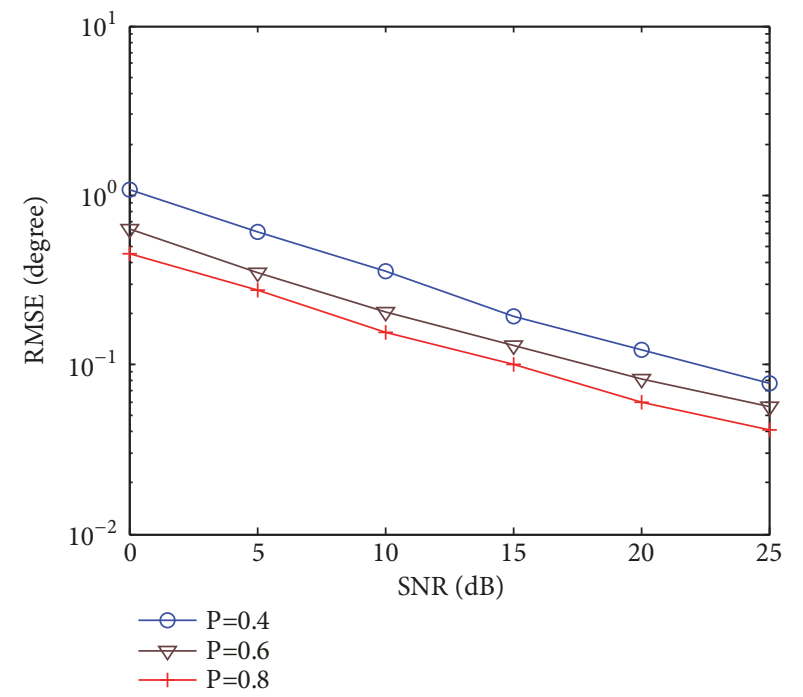

FIGURE 6: DOA estimation performance under different compression ratio.

model is $15 \times 15 \times 200$ with $P=0.4, P=0.6$, and $P=0.8$. It is shown in Figure 6 that the estimation performance of our method improves with $P$ getting larger.

Simulation 4. The angle estimation performance of the proposed algorithm versus $J$ is illustrated in Figure 7, where the rectangular array is structured as $M=10, N=10$ and $M^{\prime}=N^{\prime}=8, J^{\prime} / J=0.8$. It is verified in Figure 7 that the proposed algorithm can obtain improved DOA estimation accuracy with a larger snapshots.

Simulation 5. Figure 8 shows DOA estimation performance of the proposed algorithm with varied $M$. Assume that $N=$ $N^{\prime}=10$ and $J=J^{\prime}=200$ with $M^{\prime} / M=2 / 3$. It is attested 


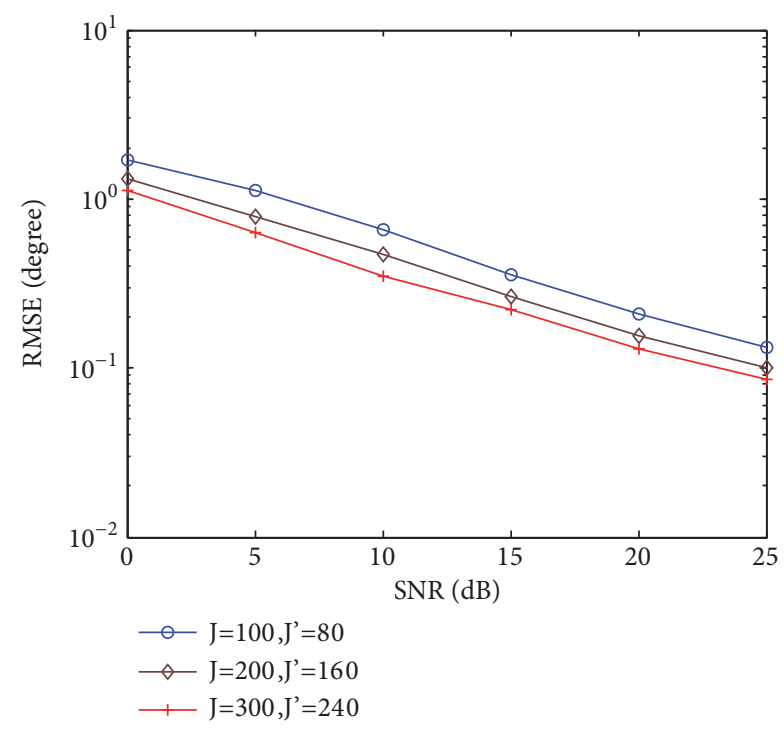

FIGURE 7: DOA estimation performance under different snapshots.

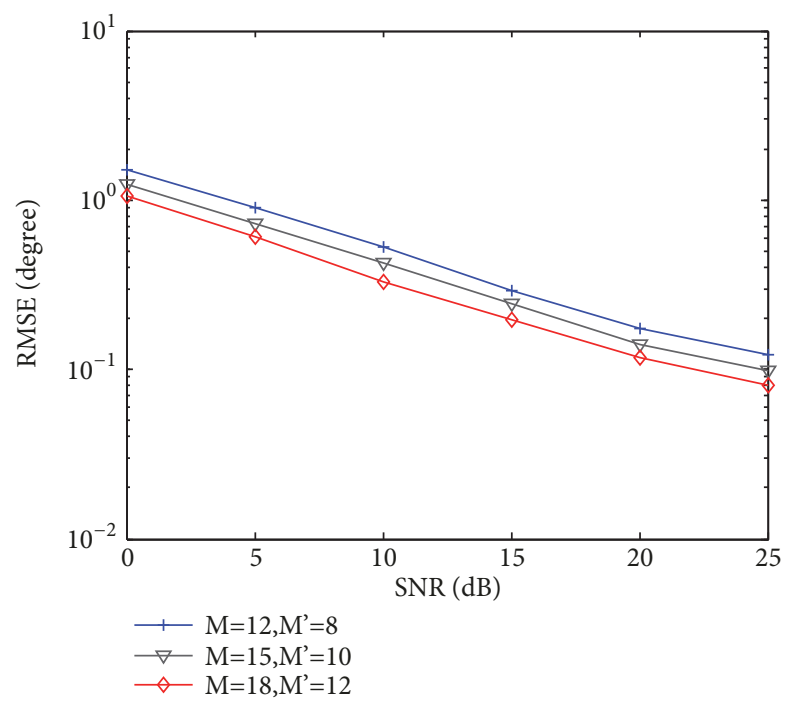

Figure 8: DOA estimation performance under different $M$.

by Figure 8 that the estimation performance of the proposed method gets better with the increasing $M$.

Simulation 6. The estimation performance of the proposed method versus $N$ is illustrated in Figure 9, where $M=$ $15, J=200$ with $M^{\prime} / M=J^{\prime} / J=N^{\prime} / N=0.8$. It is indicated obviously that the proposed method obtains improved performance with larger $N$.

\section{Conclusions}

We jointly utilize the CS theory and the PARALIND decomposition in this paper and propose a CS-PARALIND algorithm with a uniform rectangular array to extract the DOA estimates of coherent signals. The proposed algorithm can attain autopaired 2D-DOA estimates, and benefiting from

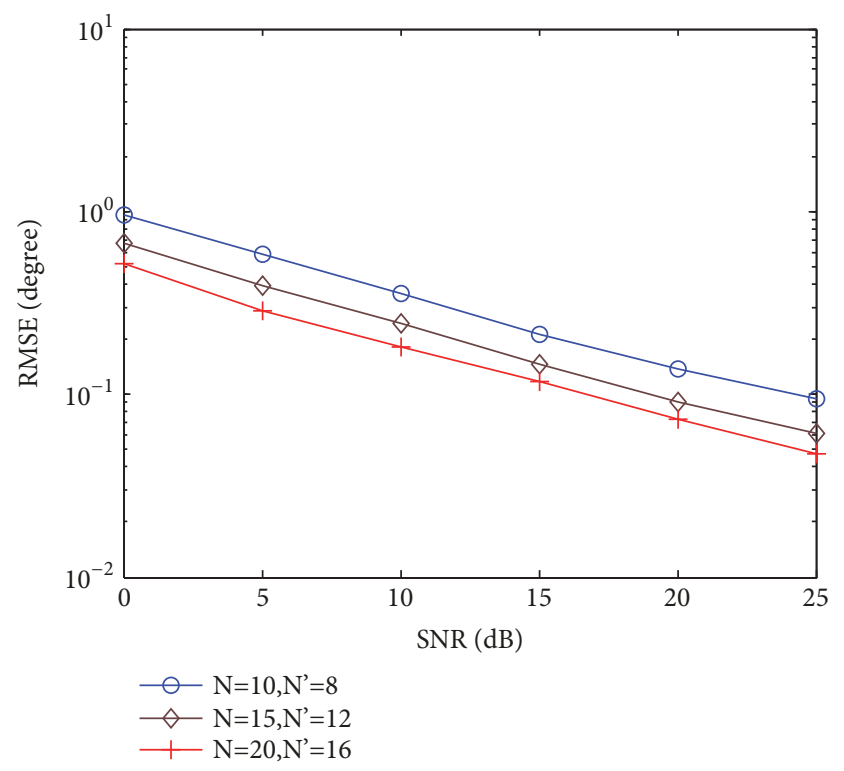

Figure 9: DOA estimation performance under different $N$.

the compression procedure, lower computational cost, and smaller demand for storage capacity can be achieved. Extensive simulations corroborate that the proposed approach obtains superior estimation performance compared to the traditional PARALIND algorithm and, significantly, outperforms the FBSS-PM and FBSS- ESPRIT algorithm.

\section{Data Availability}

The simulation data used to support the findings of this study are included within the article.

\section{Conflicts of Interest}

The authors declare that there are no conflicts of interest regarding the publication of this paper.

\section{Acknowledgments}

This work is supported by China NSF Grants (61371169, 61601167), the Fundamental Research Funds for the Central Universities (NT2019013), Graduate Innovative Base (laboratory) Open Funding of Nanjing University of Aeronautics and Astronautics (kfjj20170412), and Shandong Province NSF Grants (ZR2016FM43).

\section{References}

[1] X. Y. Yu and M. Q. Zhou, "A DOA estimation algorithm in OFDM radar-communication system for vehicular application," Advanced Materials Research, vol. 926-930, pp. 1853-1856, 2014.

[2] J. Li, X. Zhang, and H. Chen, "Improved two-dimensional DOA estimation algorithm for two-parallel uniform linear arrays using propagator method," Signal Processing, vol. 92, no. 12, pp. 3032-3038, 2012. 
[3] X. F. Zhang, R. A. Cao, and M. Zhou, "Noncircular-PARAFAC for 2D-DOA estimation of noncircular signals in arbitrarily spaced acoustic vector-sensor array subjected to unknown locations," EURASIP Journal on Advances in Signal Processing, vol. 2013, no. 1, article no. 107, 2013.

[4] X. Zhang, L. Xu, D. Xu et al., "Direction of departure (DOD) and direction of arrival (DOA) estimation in MIMO radar with reduced-dimension MUSIC, IEEE Communications Letters, vol. 14, no. 12, pp. 1161-1163, 2010.

[5] R. Roy and T. Kailath, "ESPRIT-estimation of signal parameters via rotational invariance techniques," IEEE Transactions on Signal Processing, vol. 37, no. 7, pp. 984-995, 1989.

[6] F. Gao and A. B. Gershman, "A generalized ESPRIT approach to direction-of-arrival estimation," IEEE Signal Processing Letters, vol. 12, no. 3, pp. 254-257, 2005.

[7] R. O. Schmidt, "Multiple emitter location and signal parameter estimation," IEEE Transactions on Antennas and Propagation, vol. 34, no. 3, pp. 276-280, 1986.

[8] S. Marcos, A. Marsal, and M. Benidir, "The propagator method for source bearing estimation," Signal Processing, vol. 42, no. 2, pp. 121-138, 1995.

[9] T. Filik and T. E. Tuncer, "2-D paired direction-of-arrival angle estimation with two parallel uniform linear arrays," International Journal of Innovative Computing, Information and Control, vol. 7, no. 6, pp. 3269-3279, 2011.

[10] Y.-Y. Wang and S.-C. Huang, "An ESPRIT-based algorithm for 2D-DOA estimation," IEICE Transactions on Fundamentals of Electronics, Communications and Computer Sciences, vol. E94.A, no. 9, pp. 1847-1850, 2011.

[11] C. P. Mathews, M. Haardt, and M. D. Zoltowski, "Performance analysis of closed-form, ESPRIT based 2-D angle estimator for rectangular arrays," IEEE Signal Processing Letters, vol. 3, no. 4, pp. 124-126, 1996.

[12] Y. Hua, "A pencil-MUSIC algorithm for finding twodimensional angles and polarizations using crossed dipoles," IEEE Transactions on Antennas and Propagation, vol. 41, no. 3, pp. 370-376, 1993.

[13] W. Zheng, X. Zhang, and H. Zhai, "A generalized coprime planar array geometry for two-dimensional DOA estimation," IEEE Communications Letters, vol. 99, p. 1, 2017.

[14] Y.-Y. Dong, C.-X. Dong, Y.-T. Zhu, G.-Q. Zhao, and S.-Y. Liu, "Two-dimensional DOA estimation for L-shaped array with nested subarrays without pair matching," IET Signal Processing, vol. 10, no. 9, pp. 1112-1117, 2016.

[15] T.-J. Shan, M. Wax, and T. Kailath, "On spatial smoothing for direction-of-arrival estimation of coherent signals," IEEE Transactions on Signal Processing, vol. 33, no. 4, pp. 806-811, 1985.

[16] S. U. Pillai and B. H. Kwon, "Forward/backward spatial smoothing techniques for coherent signal identification," IEEE Transactions on Signal Processing, vol. 37, no. 1, pp. 8-15, 1989.

[17] S. Qin, Y. D. Zhang, and M. G. Amin, "DOA estimation of mixed coherent and uncorrelated targets exploiting coprime MIMO radar," Digital Signal Processing, vol. 61, pp. 26-34, 2017.

[18] Y. Hu, Y. Liu, and X. Wang, "Doa estimation of coherent signals on coprime arrays exploiting fourth-order cumulants," Sensors, vol. 17, no. 4, article no. 682, 2017.

[19] J. B. Kruskal, “Three-way arrays: rank and uniqueness of trilinear decompositions, with application to arithmetic complexity and statistics," Linear Algebra and its Applications, vol. 18, no. 2, pp. 95-138, 1977.
[20] R. Cao, X. Zhang, and C. Wang, "Reduced-dimensional PARAFAC-based algorithm for joint angle and doppler frequency estimation in monostatic MIMO radar," Wireless Personal Communications, vol. 80, no. 3, pp. 1231-1249, 2014.

[21] N. D. Sidiropoulos, L. De Lathauwer, X. Fu, K. Huang, E. E. Papalexakis, and C. Faloutsos, "Tensor decomposition for signal processing and machine learning," IEEE Transactions on Signal Processing, vol. 65, no. 13, pp. 3551-3582, 2017.

[22] N. D. Sidiropoulos, R. Bro, and G. B. Giannakis, "Parallel factor analysis in sensor array processing," IEEE Transactions on Signal Processing, vol. 48, no. 8, pp. 2377-2388, 2000.

[23] R. Bro, R. A. Harshman, N. D. Sidiropoulos, and M. E. Lundy, "Modeling multi-way data with linearly dependent loadings," Journal of Chemometrics, vol. 23, no. 7-8, pp. 324-340, 2009.

[24] M. Bahram and R. Bro, "A novel strategy for solving matrix effect in three-way data using parallel profiles with linear dependencies," Analytica Chimica Acta, vol. 584, no. 2, pp. 397402, 2007.

[25] C. Chen, X. F. Zhang, and D. Ben, "Coherent angle estimation in bistatic multi-input multi-output radar using parallel profile with linear dependencies decomposition," IET Radar Sonar and Navigation, vol. 7, no. 8, pp. 867-874, 2013.

[26] X. F. Zhang, M. Zhou, and J. F. Li, "A PARALIND decomposition-based coherent two-dimensional direction of arrival estimation algorithm for acoustic vector-sensor arrays," Sensors, vol. 13, no. 4, p. 5302, 2013.

[27] D. L. Donoho, “Compressed sensing," IEEE Transactions on Information Theory, vol. 52, no. 4, pp. 1289-1306, 2006.

[28] E. J. Candes, J. Romberg, and T. Tao, "Robust uncertainty principles: exact signal reconstruction from highly incomplete frequency information," Institute of Electrical and Electronics Engineers Transactions on Information Theory, vol. 52, no. 2, pp. 489-509, 2006.

[29] D. Malioutov, M. Cetin, and A. S. Willsky, "A sparse signal reconstruction perspective for source localization with sensor arrays," IEEE Transactions on Signal Processing, vol. 53, no. 8, pp. 3010-3022, 2005.

[30] X. Yang, S. A. Shah, A. Ren et al., "Wandering pattern sensing at S-band," IEEE Journal of Biomedical and Health Informatics, vol. 22, no. 6, pp. 1863-1870, 2018.

[31] J.-Q. Wu, W. Zhu, and B. Chen, "Compressed sensing techniques for altitude estimation in multipath conditions," IEEE Transactions on Aerospace and Electronic Systems, vol. 51, no. 3, pp. 1891-1900, 2015.

[32] C.-R. Tsai and A.-Y. Wu, "Structured random compressed channel sensing for millimeter-wave large-scale antenna systems," IEEE Transactions on Signal Processing, vol. 66, no. 19, pp. 50965110, 2018.

[33] N. D. Sidiropoulos and A. Kyrillidis, "Multi-way compressed sensing for sparse low-rank tensors," IEEE Signal Processing Letters, vol. 19, no. 11, pp. 757-760, 2012.

[34] T. Nishimura, Y. Ogawa, and T. Ohgane, "DOA estimation by applying compressed sensing techniques," in Proceedings of the 2014 IEEE International Workshop on Electromagnetics: Applications and Student Innovation Competition, IEEE $i$ WEM 2014, pp. 121-122, Japan, August 2014.

[35] C. Guang and L. Qi, "Compressed sensing-based angle estimation for noncircular sources in MIMO radar," in Proceedings of the 4th International Conference on Instrumentation and Measurement, Computer, Communication and Control, IMCCC 2014, pp. 40-44, China, September 2014. 
[36] H. X. Yu, X. F. Qiu, X. F. Zhang et al., "Two-dimensional direction of arrival (DOA) estimation for rectangular array via compressive sensing trilinear model," International Journal of Antennas \& Propagation, vol. 2015, 10 pages, 2016.

[37] J. A. Tropp and A. C. Gilbert, "Signal recovery from random measurements via orthogonal matching pursuit," IEEE Transactions on Information Theory, vol. 53, no. 12, pp. 4655-4666, 2007.

[38] X. F. Zhang, F. Wang, and H. W. Chen, Theory and Application of Array Signal Processing, National Defense Industry Press, Beijing, China, 2012.

[39] S. Li and X. F. Zhang, "Study on the compressed matrices in compressed sensing trilinear model," Applied Mechanics and Materials, vol. 556-562, pp. 3380-3383, 2014.

[40] L. Xu, R. Wu, X. Zhang, and Z. Shi, "Joint two-dimensional DOA and frequency estimation for L-Shaped array via compressed sensing PARAFAC method," IEEE Access, vol. 6, pp. 37204-37213, 2018.

[41] P. Stoica and A. Nehorai, "Performance study of conditional and unconditional direction-of-arrival estimation," IEEE Transactions on Signal Processing, vol. 38, no. 10, pp. 1783-1795, 1990. 


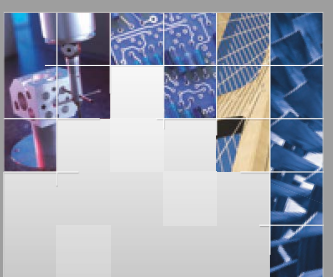

\section{Enfincering}
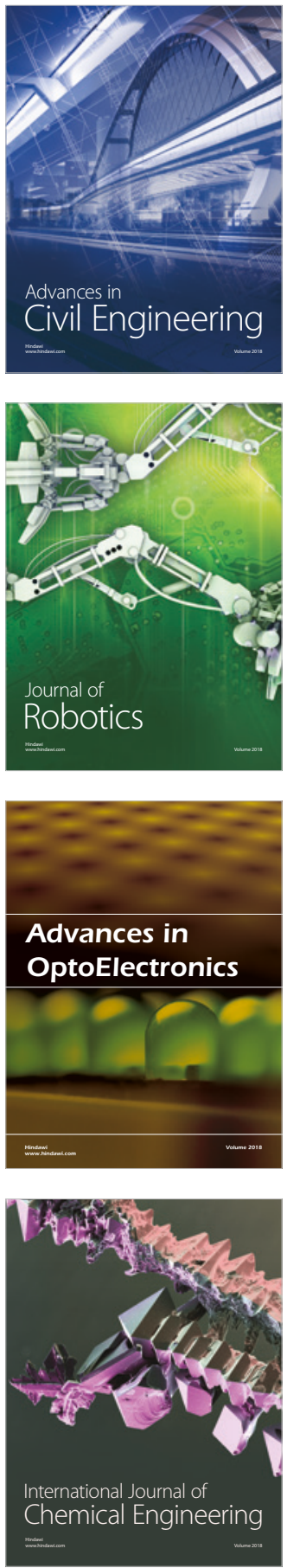

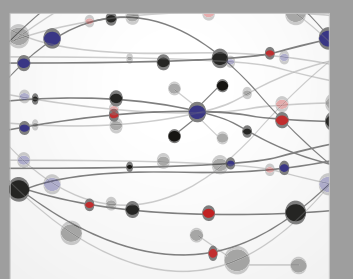

\section{Rotating \\ Machinery}

The Scientific World Journal

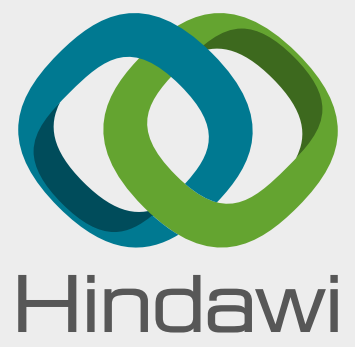

Submit your manuscripts at

www.hindawi.com
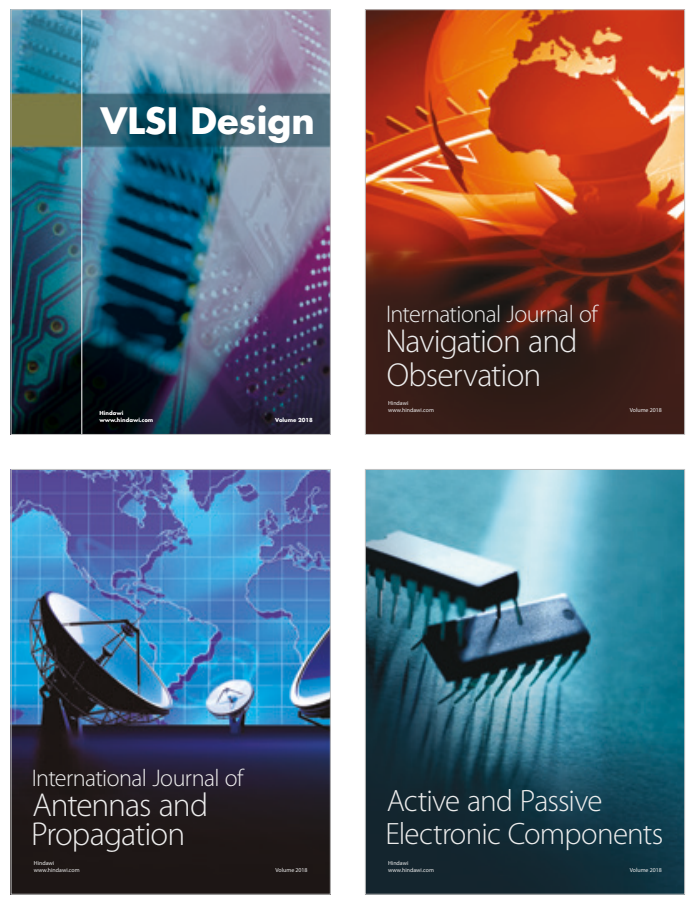
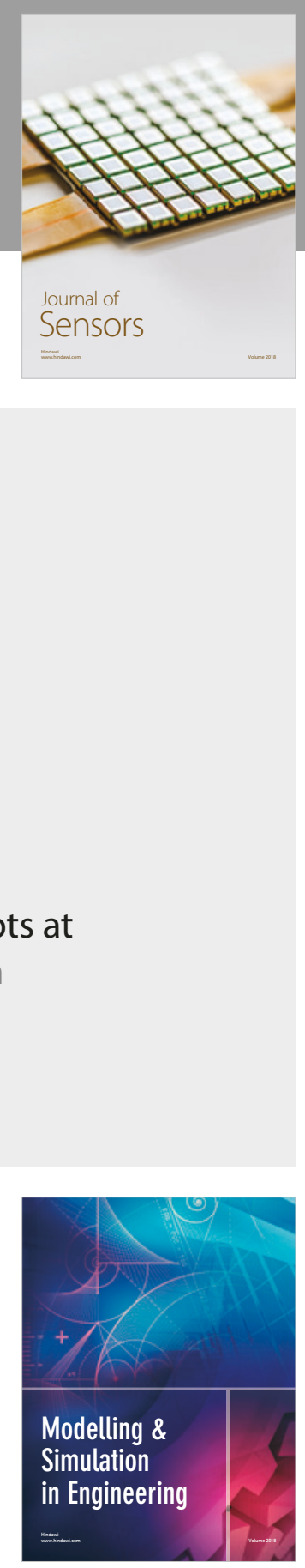

\section{Advances \\ Multimedia}
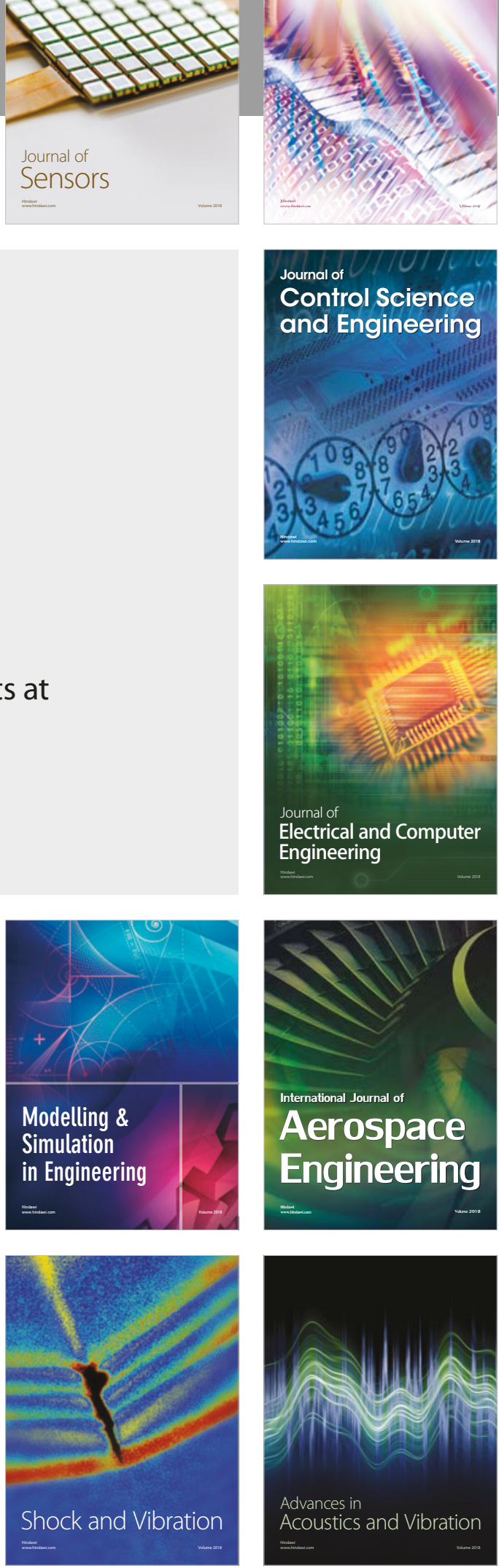